\title{
The unique impact of COVID-19 on orthopedic surgery residency applicants and program directors in Canada
}

\author{
Ajay Shah, BSc (Hons.) \\ Allen A. Champagne, $\mathrm{PhD}$ \\ Joshua Del Papa, PhD \\ Jay Toor, MD, MBA \\ Jeremie Larouche, MD \\ Markku T. Nousiainen, MD
}

Accepted February 9, 2021

\section{Correspondence to:}

A. Shah

Michael G. DeGroote School of Medicine

McMaster University

1200 Main St West

Hamilton ON L8N $3 Z 5$

ajay.shah@medportal.ca

DOI: $10.1503 /$ cjs.002021

\section{SUMMARY}

The COVID-19 pandemic led to the cancellation of visiting medical student electives and in-person residency interviews in Canada. Orthopedic surgery residency programs are now curtailed in their ability to selfpromote and select optimal applicants. Online and social media tools should be adopted to promote programs. Faculty, residents and trainees should aim to attend virtual social and program information events. Applicants should make efforts to learn about the programs by attending events and reaching out to residents, and strengthen their application through research and other activities to demonstrate their interest and commitment to the field of orthopedic surgery. These efforts may help avoid the prospect of qualified candidates being unranked by programs or residency positions being unfilled. These difficult circumstances may serve to engender collaboration and cooperation across residency programs and medical schools and lay the foundation for a thriving interconnected ecosystem of future orthopedic surgeons.
O rthopedic surgery has declined in popularity among medical students in Canada (Figure 1). Canada has the lowest number of orthopedic surgeons per capita among G7 nations (3.5 per 100000 people), but orthopedic patients experience the longest pretreatment wait times of all specialties and $33.2 \%$ of recent orthopedic residency graduates are actively looking for work. ${ }^{1}$

The World Health Organization (WHO) declared the COVID-19 global pandemic on Mar. 11, 2020. The Association of Faculties of Medicine of Canada (AFMC) cancelled all visiting medical student electives for the duration of the 2020-21 academic year. Application deadlines for the Canadian Resident Matching Service (CaRMS) were delayed to February 2021. The interview period, typically featuring social events and in-person interviews, was conducted virtually.

\section{THE PROBLEM}

\section{Cancellation of visiting electives}

The visiting elective allows both applicants and program directors to informally assess the applicant's fit for that specific learning environment. Some groups of applicants may be disproportionately affected by the changes due to COVID-19: students who became interested in orthopedic surgery later in training, those with a less competitive portfolio, and international medical graduates may be unable to level the playing field by displaying positive personality attributes on visiting electives. Though all residency programs across Canada provide excellent training in orthopedic surgery, there are slight variations in education style; institutional objectives; faculty phenotype; and clinical volume, exposure and practice 


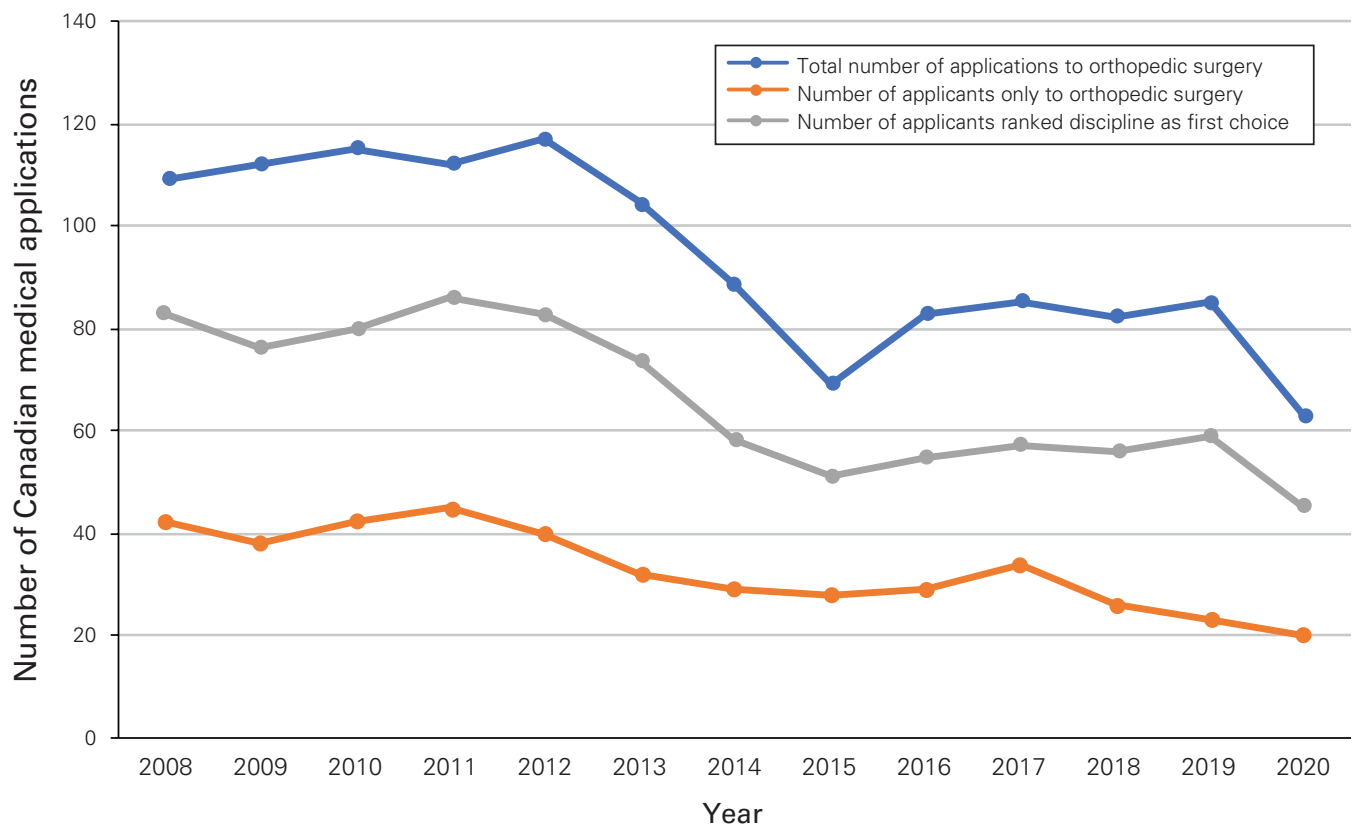

Fig. 1. Decline in popularity of orthopedic surgery as the top choice of disciplines.

Table 1. A summary of AFMC recommendations to applicants and program directors ${ }^{7}$

Applicants

Do Visit the central calendar of program promotion events and participate in those that interest you. Attend virtual presentations/learning opportunities that programs are hosting. Speak with residents from other training programs.
Program directors

Adhere to the recommendations of Best Practice of Assessment and Selection. Provide virtual opportunities for candidates to learn about your program such as virtual presentations and "town halls." Provide opportunities for candidates to get a sense of your culture by offering interactive teaching sessions and to interact with residents via virtual "fireside chats." Consider providing opportunities for virtual group discussion with the program director. Develop creative and relevant material to be placed on the centralized online portal.
Don't Do not arrange visiting electives, clinical or nonclinical experiences. Do not arrange in person site visits or one-on-one meetings with a program.
Do not arrange unsanctioned electives with learners, including observerships, virtual electives or site visits, or have "informal" in-person meetings with potential applicants. Do not invite applicants to give a special presentation at an academic day, to an audience of those who are involved in file reviews or are on the selection committee, and other similar scenarios. Do not use applicant attendance as a surrogate measure of program interest. If applicants are required to register for an event, programs should not use this information as part of the file review and ranking process. Do not place responsibilities of program promotion on a single trainee.

AFMC $=$ Association of Faculties of Medicine of Canada.

patterns that result in better or worse fits for different trainees. ${ }^{2}$ Trainees make subjective judgments of resident collegiality, program fit, resident-staff interactions, and potential red flags and weigh these in their eventual decision when ranking programs. ${ }^{3}$

\section{Impact on selection process}

Canadian file reviewers focus on subjective assessments (e.g., rotation performance, curriculum vitae, personal letters, interview performance) to select applicants. Surveys of Canadian surgical program directors have shown the importance of a rotation at the school of interest in determining a student's final rank-order position. Relying solely on interview performance can increase susceptibility to bias, which may promote "in-breeding." 4 This could be problematic for a field already criticized for a lack of racial and gender diversity. ${ }^{5}$ Both applicants and programs may feel unfairly represented in virtual interviews. ${ }^{6}$ Thus, programs may struggle to balance their mandates of ensuring social accountability and encouraging diversity.

\section{SOLUTIONS}

\section{Ongoing initiatives}

In response to the cancellation of visiting electives, the AFMC advised on best practices for virtual engagement 
Table 2. Analysis of orthopedic surgery residency programs' utilization of online and social media tools as of Nov. 23, 2020

\begin{tabular}{lc|} 
Factor & $\begin{array}{c}\text { No. of } \\
\text { programs }\end{array}$ \\
\hline $\begin{array}{l}\text { Statement to address COVID-19 pandemic's impact on } \\
\text { upcoming match posted on website }\end{array}$ & $0 / 17$ \\
\hline $\begin{array}{l}\text { Virtual program information event scheduled on AFMC online } \\
\text { calendar }\end{array}$ & $12 / 17$ \\
\hline $\begin{array}{l}\text { Resident profiles (e.g., photos, name, bio, contact info) on } \\
\text { website }\end{array}$ & $7 / 17$ \\
\hline Residency curriculum posted on website & $6 / 17$ \\
\hline Active research updates or blog postings on website & $4 / 17$ \\
\hline Active use of Instagram & $9 / 17$ \\
\hline Active use of Twitter & $2 / 17$ \\
\hline Active use of Facebook & $1 / 17$ \\
\hline AFMC = Association of Faculties of Medicine of Canada. & \\
\hline
\end{tabular}

and applicant selection (Table 1) and launched a novel online tool in November 2020. The tool is for residents, faculty and program directors to schedule upcoming events, collate links to other relevant websites and post multimedia content about the program. It may be useful as a sustainable long-term program outreach tool, particularly for smaller programs that may not attract students on electives.

An analysis of orthopedic surgery residency programs' use of online and social media tools is found in Table 2. As of Jan. 16, 2021, 12 orthopedic surgery programs had scheduled virtual program information nights. Virtual events consisted of town hall presentations and breakout rooms, with residents or faculty answering applicants' questions. Additionally, a group of medical students organized a series of virtual panel seminars featuring 12 Canadian orthopedic surgery program directors. The seminars served as an effective platform for senior Canadian medical students with an interest in orthopedic surgery to meet program directors virtually and glean insight into each participating residency program. In the panel sessions, program directors recommended that applicants seeking to increase their knowledge and visibility to programs start by emailing residents and joining virtual academic half-days or journal clubs. Applicants reported that they enjoyed breakout room sessions with residents, which allowed them to observe resident interactions. Using these seminars as a platform for further growth and collaboration among participants, the same students then launched the Canadian Orthopaedic Surgery Interest Network (COSIN) page with the objective of fostering a community of interconnected medical students and sharing learning opportunities specific to orthopedic surgery.

\section{Applicant strategies}

Students should attend virtual learning events and use online tools to learn about programs. Programs may inquire about how applicants spent their time while not in the hospital because of COVID-19, and students should be prepared to answer these questions in a meaningful way. Students may also consider contributing to volunteer efforts to help the hospital or community; examples include sewing masks or creating other personal protective equipment, participating in telehealth or COVID-19 hotlines, or assisting with medical school education. Applicants can also seek opportunities to assist with ongoing research projects. Regardless of the subject matter, research is a means for applicants to exhibit intellectual curiosity, dedication to scientific investigation, critical analysis skills, leadership capabilities, perseverance and the ability to work within a team. Programs with more of a research emphasis may preferentially consider research experience. ${ }^{8}$

\section{RECOMMENDATIONS}

\section{Programs}

Programs should host virtual events featuring presentations by faculty and breakout rooms with current residents to give applicants a feel for the culture of a program. Programs can maintain an active virtual presence using social media, videos and podcasts via the AFMC online tool to feature the unique aspects of a program and highlight the achievements of faculty and residents. Programs can encourage attendance to virtual learning events, such as daily teaching rounds and journal clubs. Programs should consider accepting letters of recommendation from community and nonorthopedic surgeons. Programs can be transparent with the selection criteria of all application components, including interviews. Virtual interviews should involve assessment by a diverse group of residents and faculty, ideally using multiple mini-interviews or several rounds of interviews.

\section{Applicants}

It is recommended that applicants attend the virtual events of multiple programs and reach out to current residents at programs of interest. Applicants should utilize the online and social media tools available to learn about different residency programs. Applicants can participate in research projects to strengthen their CaRMS applications and engage in extracurricular activities that display leadership, collaboration and altruism.

\section{An unintended benefit of COVID-19?}

One may consider that the eradication of visiting electives (estimated $\$ 2000$ per rotation) and in-person 
interviews (estimated $\$ 7000$ for interview season) offers some financial benefit to students. Future qualitative research should be directed toward assessing the phenomenological experience of applicants and program directors throughout the residency application process.

Affiliations: From the Michael DeGroote School of Medicine, Faculty of Health Sciences, McMaster University, Hamilton, Ont. (Shah); the School of Medicine, Faculty of Health Sciences, Queen's University, Kingston, Ont. (Champagne, Del Papa); and the Division of Orthopaedic Surgery, Department of Surgery, University of Toronto, Toronto, Ont. (Toor, Larouche, Nousiainen).

Competing interests: None declared.

Contributors: All authors contributed substantially to the conception, writing and revision of this article and approved the final version for publication.

Content licence: This is an Open Access article distributed in accordance with the terms of the Creative Commons Attribution (CC BY-NC-ND 4.0) licence, which permits use, distribution and reproduction in any medium, provided that the original publication is properly cited, the use is noncommercial (i.e., research or educational use), and no modifications or adaptations are made. See: https://creativecommons.org/licenses/by-nc-nd/4.0/

\section{References}

1. Unemployment and underemployment of orthopaedic surgeons. Highly trained and underemployed: Are we making any progress in our professional crisis? Canadian Orthopaedic Association; 2020. Available: https://coa-aco.org/unemployment-and-underemployment -of-orthopaedic-surgeons/ (accessed 2020 Nov. 15).

2. Mann U, Nayak JG. The potential impact of COVID-19 on the Canadian Resident Matching Service: unique future challenges faced by urology residency programs and applicants. Can Urol Assoc f 2020;14:E167-8.

3. Strelzow J, Petretta R, Broekhuyse HM. Factors affecting orthopedic residency selection: a cross-sectional survey. Can 7 Surg 2017;60:186-91.

4. Quintero AJ, Segal LS, King TS, et al. The personal interview: assessing the potential for personality similarity to bias the selection of orthopaedic residents. Acad Med 2009;84:1364-72.

5. Shah KN, Ruddell JH, Scott B, et al. Orthopaedic surgery faculty. 7B7S Open Access. 2020;5:e20.00009-e20.00009.

6. Healy WL, Bedair H. Videoconference interviews for an adult reconstruction fellowship: lessons learned. F Bone foing Surg 2017;99:E114.

7. The Association of Faculties of Medicine of Canada. Canadian Residency Virtual Promotion Guide; 2020. Available: https://www.afmc.ca/ web/sites/default/files/pdf/2020-Canadian-Residency-Virtual-Promotion -Guide_en.pdf (accessed 202 Nov. 15).

8. Campbell ST, Gupta R, Avedian RS. The effect of applicant publication volume on the orthopaedic residency match. 7 Surg Educ 2016;73:490-5. 